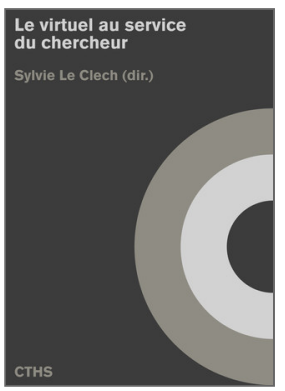

Sylvie Le Clech (dir.)

Le virtuel au service du chercheur

Éditions du Comité des travaux historiques et scientifiques

\title{
Intégrer le virtuel dans l'analyse sociologique : concepts théoriques et exemples d'approche empirique
}

\section{Ariane Richard-Bossez et Philippe Vitale}

DOI : $10.4000 /$ books.cths. 15353

Éditeur : Éditions du Comité des travaux historiques et scientifiques

Lieu d'édition : Éditions du Comité des travaux historiques et scientifiques

Année d'édition : 2021

Date de mise en ligne : 21 juin 2021

Collection: Actes des congrès nationaux des sociétés historiques et scientifiques

EAN électronique : 9782735509300

\section{Cbooks}

http://books.openedition.org

\section{Référence électronique}

RICHARD-BOSSEZ, Ariane ; VITALE, Philippe. Intégrer le virtuel dans l'analyse sociologique : concepts théoriques et exemples d'approche empirique In : Le virtuel au service du chercheur [en ligne]. Paris : Éditions du Comité des travaux historiques et scientifiques, 2021 (généré le 24 juin 2021). Disponible sur Internet : <http://books.openedition.org/cths/15353>. ISBN : 9782735509300. DOI : https:// doi.org/10.4000/books.cths. 15353

Ce document a été généré automatiquement le 24 juin 2021. 


\title{
Intégrer le virtuel dans l'analyse sociologique : concepts théoriques et exemples d'approche empirique
}

\author{
Ariane Richard-Bossez et Philippe Vitale
}

1 Les sciences sociales, et parmi elles la sociologie, se considèrent habituellement comme des sciences de description et d'analyse du réel ${ }^{1}$. Cependant, la nature de ce réel reste bien souvent peu interrogée. Nous proposons dans cet article une réflexion autour de la notion de virtuel et de ses rapports au réel permettant, nous semble-t-il, d'enrichir l'analyse sociologique. Cette approche se nourrit de différents travaux menés au sein du Laboratoire méditerranéen de sociologie (UMR 7305, Aix-Marseille Université/ CNRS), et notamment de ceux de Nicole Ramognino².

2 Le propos se déclinera en trois temps. Nous commencerons par exposer les concepts théoriques sur lesquels nous nous appuyons, en particulier ceux développés par les philosophes Pierre Livet et Frédéric Nef. Puis nous illustrerons cette approche à partir de deux recherches touchant à deux phénomènes très différents. D'une part, une analyse de l'interprétation de l'insurrection des esclaves de 1811 à La Réunion ${ }^{3}$; d'autre part, une recherche sur le processus de construction des savoirs et ses inégalités à l'école maternelle ${ }^{4}$. Enfin, la conclusion reviendra sur l'intérêt d'une telle approche pour le travail sociologique et, plus globalement, pour les sciences sociales.

\section{Intégrer les virtualités à l'analyse sociologique : ancrages théoriques}

3 Notre entrée sociologique part du principe qu'il est nécessaire de se poser des questions ontologiques sur les objets que nous observons avant de les analyser. À la suite des philosophes Pierre Livet et Frédéric Nef, nous considérons que les entités de base du social sont des processus ${ }^{5}$, car «tout fait social implique la poursuite de dynamiques 
sociales $^{6} »$. Il s'agit dès lors d'interroger la nature de ces processus sociaux et d'en proposer une « ontologie $e^{7}$.

Pour ces auteurs, tout processus est une transformation d'un aspect en un autre et se fonde sur un couplage entre une existence actuelle (réalisée) et une existence virtuelle (potentielle) :

«Un processus est un flux : quand il se déploie, quand il entre dans l'actualité, son accomplissement est virtuel, et quand son accomplissement s'actualise son démarrage est devenu virtuel ${ }^{8}$.»

5 Ainsi, si je prête un objet à quelqu'un, au moment du prêt, le retour de cet objet reste virtuel et pourrait ne pas advenir. Quand cet objet m'est rendu, c'est son existence au moment du prêt qui est devenue virtuelle, et l'objet tel qu'il m'est restitué possède une existence différente de celle qu'il avait quand je l'ai prêté.

6 Mais dans ce couplage, il existe des "substituabilités " ", c'est-à-dire que l'accomplissement d'un processus reste ouvert tant qu'il n'est pas réalisé, et qu'il peut prendre des chemins divers. De la sorte, dans l'exemple du prêt d'un objet, entre le moment où je prête cet objet et celui où il m'est rendu, différentes options sont possibles : l'objet peut être usé, abimé, cassé, prêté à une tierce personne, réparé, perdu, remplacé... Ces éventualités sont autant de substituabilités qui peuvent se présenter au cours du processus de prêt. Cet éventail d'activités substituables permet le maintien du processus et son efficience, puisqu'il rend possible le remplacement des activités par d'autres quand celles-ci ne peuvent pas s'actualiser.

7 Ainsi, il existe toujours des marges de manœuvre dans un processus, ce que les auteurs appellent du " vague ${ }^{10}$ ». Ce vague n'est cependant pas absolu. Pierre Livet et Frédéric Nef distinguent ainsi l'actuel qui correspond à ce qui existe effectivement, le possible qui n'existe pas mais pourrait exister, et le virtuel qu'ils définissent comme un possible réalisable, susceptible d'avoir un effet sur le réel. Ainsi, pour ces auteurs, «la virtualité diffère de la possibilité en ce sens que le possible n'a pas tendance à passer à la réalité $^{11}{ }$. En effet, tout processus est soumis à des contraintes, que celles-ci soient liées aux capacités des êtres humains, à l'environnement dans lequel ils évoluent ou aux interactions qu'ils nouent avec leurs pairs, d'autres êtres vivants ou objets.

Les auteurs proposent dès lors aux sociologues de viser une " enquête ontologique ${ }^{12}$ ", c'est-à-dire de « distinguer les dynamiques dont les ressorts sont efficaces et celles qui ne le sont pas $^{13}$ ». Il convient alors de rechercher ce qui permet à certaines de ces virtualités de se réaliser tandis que d'autres sont mises en suspens, voire closes. Dans ces processus, les institutions sociales - comme la famille, les administrations, les entreprises, l'école, l'armée... - jouent un rôle essentiel puisque :

«Une institution dote ses membres d'une part d'un potentiel de relations virtuelles très complexes et sophistiquées et d'autre part de ces procédés qui permettent, en appuyant les activités effectives sur ces relations virtuelles, de donner aux activités effectivement réalisées plus d'efficace et de liens avec d'autres activités qu'elles ne pourraient en avoir sans cela ${ }^{14}$.

9 Le cadre social dans lequel se développe une activité permet à la fois à certains virtuels de s'exprimer et limite les possibles. Le langage, autre institution sociale s'il en est, participe également de ces processus :

«Le langage permet ainsi à la fois de créer des ponts virtuels entre activités, et de couper d'autres ponts, si bien qu'il façonne le réseau des activités sociales. Le langage permet de ramener certains virtuels au rang de simples possibles ${ }^{15}$.» 
Observer un processus à l'aune de cette approche permet ainsi de faire ressortir ce qui a permis sa réalisation, mais également les virtualités qui ont été closes et qui généralement restent invisibles à l'analyse. En effet, se centrer sur la réalisation actuelle d'un processus peut conduire à ne rechercher que les seules substituabilités ayant conduit à cette actualisation, en oubliant d'autres substituabilités qui sont restées virtuelles au cours de son déroulement, mais potentiellement réalisables. En ce sens, cette approche permet une forme de sociologie critique en mettant en lumière des virtualités potentiellement réalisables et en pointant ce qui en empêche la réalisation. C'est cette approche heuristique que les deux parties de cet article tenteront de mettre en évidence.

\section{L'exemple de l'insurrection des esclaves de 1811 à l'île de la Réunion}

Historiens comme sociologues sont bien en difficulté épistémologique face à l'oralité, surtout quand les sources sont rares, voire inexistantes, et que leurs interprétations divergent. Face aux entretiens, les chercheurs en sciences sociales n'auraient d'autres choix que d'opter pour des théories de la conscience ou de la non-conscience des locuteurs. Une fois le camp choisi entre « ce qui se fait » et ce qui est « dit de ce qui est fait ", les entretiens revêtiraient tantôt une valeur probatoire, objectivée, une certaine réalité, tantôt une valeur exemplaire, subjective, mais supposée exprimer mieux que toute autre le vécu des/par les individus. Reste alors en suspens la question du vrai et du faux de la parole des individus à propos d'événements ou de pratiques, notamment passées. Cette tension ne se pose généralement pas pour le sociologue qui pense posséder les clés de la compréhension et de l'usage de l'entretien, protégé par une rupture bachelardienne - le savant contre le sens commun ou avec le sujet pensant, ce qui revient mutatis mutandis à la même chose dans les analyses - qui concerne tout autant la logique restitutive qu'illustrative des entretiens ${ }^{16}$. Dans un même élan d'assurance sociologique, la vigilance accordée aux conditions des cadres de l'entretien et aux interprétations des dires - ladite approche pragmatique - n'est le plus souvent qu'un vernis sociologique qui néglige la question du statut épistémologique et linguistique de la parole des gens. La situation n'est pas meilleure, si ce n'est pire, dans les autres disciplines des sciences humaines et sociales. Dans cet espace, les historiens occupent une place un peu particulière. L'histoire a longtemps été attachée à une forme de naturalisme du temps opposant " l'avant et l'après ${ }^{17}$ ", renforcé par un réalisme naïf face à la vérité des sources et des témoignages analysés et critiqués. Mais depuis les Annales, elle a tenté de surmonter la clôture de l'historicisme et du relativisme par une historiographie qui n'a cessé de se développer à mesure qu'apparaissaient de nouveaux domaines et objets d'étude. Toutefois, comme l'ont montré notamment Reinhart Koselleck et Paul Veyne ${ }^{18}$, l'historiographie n'a pas résolu le dilemme des historiens : produire des énoncés vrais, ou selon l'épistémologie poppérienne aujourd'hui convoquée, des énoncés "non faux pour le moment », à partir de sources orales, de témoignages, de récits. Pour Reinhart Koselleck, ce dilemme est lié à l'absence d'une véritable théorie de la connaissance, seule à même de permettre de penser la question de l'objectivité, de la représentation de l'événement et de la structure, de la multiplicité des temps et des échelles, de la mémoire individuelle et collective, de la valeur des entretiens et de la parole des gens... Reinhart Koselleck souligne que les historiens 
confrontés à des sources plurielles, fragiles et oralisées, oscillent entre trois postures qui ignorent les théories de la connaissance et l'épistémologie ${ }^{19}:$ l'histoire miroir où l'historien n'est là que pour aider à mettre au jour le reflet du réel-passé; la vérité toute nue, seconde version de la métaphore du miroir, pour laquelle l'historien n'est pas invisible dans le reflet de l'histoire mais où il concentre tous ses efforts pour effacer son interprétation, son récit, afin de restituer « froidement " les événements; et enfin l'histoire libérée, au sens où la garantie de l'objectivité est l'impartialité de l'historien autonome, libre de toutes pressions politique, sociale ou psychologique.

Faisant l'hypothèse que l'oralité est le résultat d'une activité, d'un récit, j'ai laissé de côté l'aporie de la dialectique du vrai et du faux. Dans le cadre d'une recherche sur la réception de l'insurrection des esclaves de 1811 à l'île de La Réunion, et de la controverse historique et politique concernant le meneur de l'insurrection - les esclaves Élie ou Gilles ? Le Noir ou le Blanc? - et le traitre - l'esclave Figaro ? -, j’ai tenté de repérer du virtuel dans les sources orales. L'objectif fut de rechercher la genèse des possibles, des clôtures et des solutions du passé, de l'analyse de sa recomposition au présent, des diverses voies, du temps et des espaces, qui traversent les mémoires collectives récitées dans les entretiens. Par exemple, j'ai travaillé sur un entretien réalisé par l'historien Hubert Gerbeau ${ }^{20}$ en 1972 au Tévelave, commune du sud-ouest de l'île de la Réunion, auprès d'un interviewé alors âgé de 68 ans, qui n'est ni un historien ni un folkloriste, plutôt un quidam passionné du passé de son île. Fort des traditions orales de son île, l'interviewé confond cependant dans un même récit - et c'est là tout l'intérêt de cet exemple - des données historiques - individus, temps, espaces, action - et des mythes. Cela m'a conduit à proposer une série d'hypothèses sur l'insurrection de 1811, de "compossibles ${ }^{21}$ " selon les termes de Paul Veyne, de virtualités aux antipodes des canevas positivistes de l'histoire, et ce sans pour autant tomber dans le subjectivisme. Cette façon d'envisager l'entretien m'a permis de mettre en relief les enjeux mémoriels autour de l'interprétation de cette insurrection. Le syncrétisme des complots - révolte de 1811, Antilles (1831), Haïti (1791), Southampton (Virginie, 1831)... - qui apparaît dans l'interview permet de souligner également combien le récit d'une mémoire collective recontextualise le symbolique et la lutte sociale - culturelle, identitaire et mémorielle. Considérant que la valeur du témoignage importe alors d'autant moins que les logiques du récit, j'ai identifié une série d'hypothèses sur les recompositions historiques - et non plus les erreurs - de l'interviewé pour conclure sur la virtualité de ses dires. Inconsciemment ou consciemment - peu importe finalement -, le locuteur raconte la lutte commune des esclaves de La Réunion, des Antilles, d'Haïti et d'ailleurs, dans le but d'abolir les catégories du récit national et du pouvoir. Comme si ces esclaves avaient finalement franchi les frontières de l'histoire pour s'unir dans un combat mythique contre les maitres, le colonialisme et le pouvoir. Cette virtualité devient alors exemplaire d'une mémoire collective qui s'actualise au présent, selon des logiques que l'historien comme le sociologue, gagneraient à convoquer et à analyser. 


\section{L'exemple de la construction des savoirs et de ses inégalités à l'école maternelle (A. Richard-Bossez, « La construction sociale et cognitive des savoirs à l'école maternelle [...] »)}

13 Notre second exemple de la prise en compte du virtuel dans l'analyse sociologique porte sur un objet radicalement différent : la construction des savoirs et ses inégalités à l'école maternelle. Rappelons que la question des inégalités à l'école maternelle apparaît dans les travaux sociologiques à partir des années 1970, et donne lieu à différentes approches. Les unes, quantitatives, cherchent avant tout à mesurer l'étendue de ces inégalités. On sait ainsi qu'actuellement à l'école maternelle, « un tiers des élèves issus d'un milieu défavorisé (ouvrier et inactif) font partie des $10 \%$ d'élèves ayant eu le moins bon score aux évaluations", alors que c'est le cas de "moins d'un élève sur dix de milieu très favorisé (cadre ou enseignant) ${ }^{22} »$. Ces analyses quantitatives permettent de mettre en évidence le poids de l'origine sociale sur la réussite scolaire dès les premières années de scolarisation, mais laissent dans l'ombre les processus qui donnent lieu à ces inégalités. Un autre type d'approche se centre sur les écarts d'orientation culturelle entre familles et école, et montre que les pédagogies mises en place à ce premier échelon du système éducatif se rapprochent davantage des usages des classes moyennes et supérieures que de celles des classes populaires, favorisant ainsi les enfants issus de ces milieux. Ces travaux permettent ainsi de mieux saisir les relations entre école et familles, mais ne questionnent pas directement ce qui se passe à l'intérieur des classes. Enfin, c'est à l'étude des pratiques scolaires dans les classes d'école maternelle que se consacre un dernier type de travaux. Ceux-ci permettent d'approcher les processus qui, au sein des classes, participent au fait qu'une grande partie des élèves de milieu populaire ne parvient pas à entrer dans les savoirs visés par l'institution scolaire.

14 C'est dans la lignée de cette dernière approche que j'ai mené une recherche doctorale ${ }^{23}$ portant sur le processus de construction des savoirs scolaires à l'école maternelle et sur les inégalités qui s'y produisent. Ce faisant, j'ai souhaité me placer dans une approche générative des inégalités scolaires. En m'appuyant sur les propositions de Pierre Livet et Frédéric Nef (voir infra) je me suis attachée à rechercher les aspects actuels du processus de construction des savoirs à l'école maternelle, mais aussi ses aspects virtuels. J'ai ainsi tenté de saisir comment une virtualité particulière de ce processus en l'occurrence la construction inégale des savoirs dans les classes de maternelle s'actualise en empêchant d'autres virtualités de s'exprimer. En effet, on peut observer au sein des classes des moments où des apprentissages ont lieu, ou du moins s'amorcent, y compris pour les élèves les plus en difficulté. Mais ces moments demeurent ponctuels et restent de l'ordre du virtuel sans s'actualiser complètement. L'analyse peut ainsi rechercher les éléments qui empêchent ou limitent leur actualisation et ainsi mieux comprendre la genèse des inégalités scolaires. L'intérêt de cette forme d'approche critique est de ne pas prendre en compte uniquement les récurrences, mais de faire également apparaître des événements singuliers et ponctuels, révélateurs de processus à même d'éclairer le réel et toutes ses virtualités ${ }^{24}$. Si l'actualisation des processus inégalitaires semble majoritaire, il n'en reste donc pas moins que cette actualisation ne constitue pas «tout le réel». Observer le processus de construction des savoirs à l'aune de cette approche permet ainsi d'en déplier les 
dimensions, qui restent bien souvent peu prises en compte dans les analyses sociologiques.

La prise en compte des virtualités dans mon analyse s'est faite à deux niveaux. D'une part, cela m'a permis d'analyser le cadre institutionnel actuel de l'école maternelle comme un réalisé parmi d'autres virtualités. J'ai ainsi étudié les contenus et la mise en place des programmes actuels, en les comparant à ceux d'autres systèmes préscolaires, notamment européens, et d'autres périodes historiques en France depuis la fin des années 1970. On peut ainsi considérer que le modèle actuel de l'école maternelle française est un modèle qui, plus que d'autres, met l'accent sur la dimension scolaire du développement de l'enfant, prenant moins en compte les aspects de soin et d'approche éducative plus globale développés dans d'autres pays européens ou occidentaux. Cette tendance s'est encore accentuée au cours des années 1980, avec la montée en puissance de la thématique de l'échec scolaire et du rôle de préparation aux apprentissages ultérieurs qui est alors dévolu à l'école maternelle. L'analyse permet ainsi de montrer comment ce modèle s'est progressivement imposé, par le biais de certains acteurs au sein du champ pédagogique, au détriment d'autres choix potentiels.

D'autre part, j'ai mené une enquête ethnographique par observation dans six classes de grande section situées dans des milieux sociaux contrastés d'une ville moyenne du Sud de la France: trois classes en éducation prioritaire et trois classes ordinaires. Pour l'analyse de ces observations, la prise en compte du virtuel m'a amenée à percevoir des moments où des apprentissages ont lieu pour les élèves les plus en difficulté. Ces moments, même s'ils ne s'actualisent pas complètement ou pas du tout, et restent ainsi de l'ordre du virtuel, n'en demeurent pas moins une des substituabilités de la situation. Intégrer ces processus d'ouverture des apprentissages pour les élèves en difficulté, m'a permis de rechercher les éléments qui en empêchent ou en limitent l'actualisation. Plusieurs facteurs peuvent permettre de dépasser la difficulté à laquelle l'élève est confronté ou, au contraire, l'y maintenir. Parmi ces facteurs, on peut citer : l'activité elle-même - sa complexité et sa présentation plus ou moins explicite -, les apprentissages qui peuvent être réalisés en famille, mais aussi, surtout, les modalités d'interactions entre l'enseignant et l'élève. Pour illustrer ce dernier point, je m'appuierai sur deux exemples mettant en scène des élèves considérés en difficulté qui ne parviennent pas à réaliser les tâches demandées, ne maîtrisant pas les savoirs attendus. L'issue du processus est différente pour chacun. Prenons tout d'abord l'exemple de Kader : il ne parvient pas à distinguer les différentes graphies des mots reproduits sur sa fiche d'exercices et regarde celles de ses voisins. Ne sachant manifestement pas quoi faire, il joue avec ses crayons de couleur. La maitresse lui demande de lui montrer les mots correspondants, mais les réponses de Kader sont incorrectes. L'enseignante quitte l'atelier en maugréant. Kader regarde alors sa feuille en se tordant les mains, visiblement mal à l'aise.

Dans cette situation, la manière dont l'activité se déroule et les modalités d'interventions de l'enseignante ne permettent pas à cet élève de surmonter les obstacles qui l'empêchent de fournir la réponse attendue. A contrario, un autre élève, Samir, qui se trouve au départ dans une situation similaire va pouvoir, lui, dépasser les difficultés qu'il rencontre :

Enseignante : « Où est-ce que tu as [l'étiquette où est écrit] "Kora" ? »

Samir, montrant «Emmée » : « Euh »

Enseignante: "T'es sûr que c'est “Kora"? Qu'est-ce que tu entends dans

"Kooora"?» 


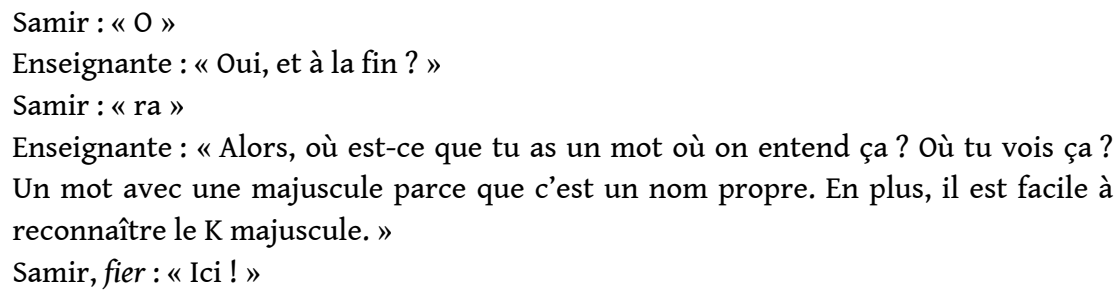

Dans ce second cas, l'intervention de l'enseignante semble permettre à l'élève de réviser ses savoirs et dépasser sa difficulté, notamment en donnant des indices basés sur des critères cognitifs qui aident Samir à retrouver dans ses étiquettes le mot "Kora». Ces deux exemples illustrent le fait qu'une même situation de difficulté scolaire peut trouver des issues différentes, qui constituent donc des virtualités du processus de construction des savoirs scolaires. Cela amène ainsi à considérer les inégalités comme résultant de la reproduction d'opérations qui ferment des possibilités d'apprentissages pour certains élèves - notamment pour ceux qui ne disposent pas déjà de certains acquis - et qui ne permettent pas d'ouverture des apprentissages autrement que de manière ponctuelle. La prise en compte du virtuel permet ainsi de proposer une approche plus générative des inégalités scolaires.

19 La confrontation de ces deux exemples de prise en compte du virtuel dans l'analyse sociologique, s'appuyant sur des méthodologies et des objets distincts - l'analyse de l'insurrection des esclaves de 1811 à l'île de la Réunion à partir d'entretiens, et l'analyse de la construction des savoirs dans l'école maternelle contemporaine à partir d'observations -, nous semble susceptible de faire d'autant mieux ressortir les apports d'une telle approche. Cela permet en effet de mettre en évidence dans chaque cas des dimensions de la situation qui ne seraient pas prises en compte par des approches où seul le réalisé serait objet. Ainsi, dans le cas de l'entretien effectué par l'historien Hubert Gerbeau, l'analyse de Philippe Vitale fait émerger des virtualités ouvrant sur de nouvelles interprétations des rapports de pouvoir entre esclaves et maîtres, qui participent à la mémoire collective et irriguent les rapports sociaux actuels. Dans le cas des inégalités de construction des savoirs à l'école maternelle, le virtuel permet de faire apparaître des moments d'ouverture des apprentissages, qu'une analyse centrée sur l'issue du processus risquerait de faire oublier. En cela, tenir compte du virtuel permet de déplier des dimensions du réel généralement peu prises en compte. In fine, c'est le changement social qui est l'horizon de cette approche, puisque le virtuel permet de saisir non seulement la genèse de l'existant mais aussi d'envisager des modes de révision de ces processus, en dévoilant les autres virtualités présentes dans le réel. 


\section{BIBLIOGRAPHIE}

ABDOUNI Sarah, « En forte baisse depuis trente ans, le retard à l'entrée en CE2 reste très dépendant du milieu social de l'élève : comparaison des panels 1978, 1997 et 2011 ", Note d'information, n² 23, Paris, Direction de l'évaluation, de la prospective et de la performance, 2015.

DEMAZIÈRE Didier et DUBAR Claude, Analyser les entretiens biographiques, Paris, Nathan, 1997.

GERBEAU Hubert, «D'une révolte à l'autre ou les deux rives de la mémoire », dans Auguste

Lacaussade (1815-1897) : poète des deux rives, Prosper Eve (éd.), Saint-André (La Réunion), Océan

Éditions, 2008.

GRANGER Gilles-Gaston, Le probable, le possible et le virtuel, Paris, Odile Jacob, 1995.

HENRI-PANABIÈRE Gaëlle, Des « héritiers » en échec scolaire, Paris, La Dispute, 2010.

KOSELLECK Reinhart, Le futur passé : contribution à la sémantique des temps historiques, Paris, Éditions de l'EHESS, 1990.

LAURENS Jean-Paul, 1 sur 500 : la réussite scolaire en milieu populaire, Toulouse, Presses universitaires du Midi, 1992.

LIVET Pierre et NEF Frédéric, Les êtres sociaux : processus et virtualité, Paris, Éditions Hermann, 2009.

PASSERON Jean-Claude, Le raisonnement sociologique, Paris, Nathan, 1991.

RAMOGNINO Nicole, « Le virtuel et sa nécessité dans l'analyse sociologique : discussion de l'ouvrage d'André Petitat, Le réel et le virtuel : genèse de la compréhension, genèse de l'action, Genève, Librairie Droz, 2009 ", SociologieS, éd. numérique, 27 janvier 2012. [URL : https://journals.openedition.org/ sociologies/3769]

RICHARD-BOSSEZ Ariane, «La construction sociale et cognitive des savoirs à l'école maternelle : entre processus différenciateurs et moments de démocratisation. Le cas des activités relatives à l'écrit en grande section ", thèse de doctorat en sociologie, Aix-en-Provence, Aix-Marseille Université, 2015 (2 vol.).

VEYNE Paul, L'inventaire des différences, Paris, Le Seuil, 1976.

VITALE Philippe, «L'aporie du vrai et du faux : le sociologue devant une approche de l'oralité par l'historien Hubert Gerbeau ", dans Pagès Michèle, Ramognino Nicole et Soldini Fabienne (dir.), L'énigme de la valeur, Aix-en-Provence, Presses universitaires de Provence, 2010.

\section{NOTES}

1. J.-C. Passeron, Le raisonnement sociologique, p. 71-88.

2. Voir par exemple N. Ramognino, « Le virtuel et sa nécessité [...]».

3. P. Vitale, « L'aporie du vrai et du faux [...]», p. 183-197.

4. A. Richard-Bossez, «La construction sociale et cognitive des savoirs à l'école maternelle [...]».

5. P. Livet et F. Nef, Les êtres sociaux [...], p. 9.

6. Ibid., p. 13. 
7. Ibid., p. 7.

8. Ibid., p. 158.

9. Ibid., p. 165.

10. P. Livet et F. Nef, Les êtres sociaux [...].

11. Ibid., p. 156. Notons que ces définitions de l'actuel, du possible et du virtuel, se distinguent de celles proposées par Gilles-Gaston Granger, voir G.-G. Granger, Le probable, le possible et le virtuel. Pour ce dernier, le virtuel n'entretient pas de rapport avec le réel, alors que le possible oui et plus encore le probable (voir Ibid., p. 13-14).

12. P. Livet et F. Nef, Les êtres sociaux [...], p. 22.

13. Ibid., p. 13.

14. Ibid., p. 168.

15. Ibid., p. 309.

16. D. Demazière et C. Dubar, Analyser les entretiens biographiques, p. 16-29.

17. R. Koselleck, Le futur passé [...], p. 133-144.

18. P. Veyne, L'inventaire des différences.

19. R. Koselleck, Le futur passé [...], p. 159-187.

20. H. Gerbeau, « D’une révolte à l'autre [...] », p. 121-139.

21. P. Veyne, L'inventaire des différences, p. 78-79.

22. S. Abdouni, «En forte baisse depuis trente ans, le retard à l'entrée en CE2 reste très dépendant du milieu social de l'élève [...] », p. 3.

23. A. Richard-Bossez, « La construction sociale et cognitive des savoirs à l'école maternelle [...]».

24. On peut rapprocher cette idée des travaux sur les "réussites improbables » telles que celles relatées par J.-P. Laurens dans son ouvrage 1 sur 500 [...]; ou des "échecs improbables » présentés par G. Henri-Panabière dans Des « héritiers » en échec scolaire.

\section{RÉSUMÉS}

Nous proposons ici une approche sociologique intégrant le virtuel sur la base des travaux des philosophes Pierre Livet et Frédéric Nef. Nous commencerons par exposer les concepts théoriques sur lesquels nous nous appuyons. Puis, nous illustrerons très brièvement le propos par deux recherches: l'une sur une analyse de l'interprétation de l'insurrection des esclaves de 1811 à La Réunion, réalisée par Philippe Vitale ; l'autre sur la construction des savoirs et ses inégalités à l'école maternelle, travaillée par Ariane Richard-Bossez. 


\section{AUTEURS}

\section{ARIANE RICHARD-BOSSEZ}

Centre méditerranéen de sociologie, de science politique et d'histoire (MESOPOLHIS, UMR 7064), Aix-Marseille Université/CNRS

PHILIPPE VITALE

Laboratoire d'économie et de sociologie du travail (LEST, UMR 7317), Aix-Marseille Université/ CNRS 